\title{
Microtubule-based Filopodium-like Protrusions Form after Axotomy
}

\author{
Daniel J. Goldberg and Donald W. Burmeister \\ Department of Pharmacology and Center for Neurobiology and Behavior, Columbia University, New York, New York \\ 10032
}

The growth cone at the front of a growing neurite often has F-actin-rich structures-digitate filopodia and sheet-like veils and lamellipodia-whose protrusion advances the leading edge. Microtubules and other cytoplasmic constituents later fill the protruded area, transforming it into new neuritic length. Growth can be initiated from an axon by transecting it. We have used video-enhanced contrast-differential interference contrast microscopy to observe the early events following transection of Aplysia axons in culture. Many filopodium-like protrusions (FLPs) grew rapidly (average instantaneous velocity of $1.6 \mu \mathrm{m} / \mathrm{sec}$ ) from the sides and end of the axon stump within minutes of transection. Some of these displayed bidirectional transport of swellings, at a rate similar to fast axonal transport. Dihydrocytochalasin B, which blocks actin polymerization, only halved the number of FLPs that formed within $\mathbf{1 0}$ min of transection, and actually increased the number of transporting FLPs. Nocodazole, a microtubule-specific drug, also halved the number of FLPs, but none of them displayed transport of swellings. No FLPs formed in the presence of both drugs. In transected axons that had not been exposed to either drug, removal of the plasma membrane revealed fibers in many of the FLPs; immunofluorescence showed these fibers to be microtubules. Thus, a substantial number of the FLPs that form soon after axotomy are microtubule based, rather than actin based, underscoring the potential of microtubules to drive the rapid extension of neuritic precursors.

The leading edge of a growing neuronal process often advances in the form of actin-rich protrusions. Digitate filopodia, which contain bundles of actin filaments, and sheet-like veils and lamellipodia, which contain meshworks of actin filaments, project rapidly from growth cones (Letourneau and Ressler, 1983; Goldberg and Burmeister, 1989). At least for filopodia, protrusion is likeliest to be driven by polymerization of actin (Smith, 1988). The subsequent entry of microtubules and membranous organelles into some of these protrusions starts to transform them into neurite and increases their stability (Goldberg and Burmeister, 1986, 1989; Aletta and Greene, 1988; Sabry et al., 1991). Selective entry of microtubules into one of several filopodia may be a mechanism of pathway selection for axons growing in a complex environment (Sabry et al., 1991).

Received Mar. 10, 1992; revised June 5, 1992; accepted June 30, 1992

We are grateful to Ms. AnnMarie Coriano and Ms. Yvette Francis for excellent technical assistance. This work was supported by NIH Research Grant NS25161.

Correspondence should be addressed to Daniel J. Goldberg, Department of Pharmacology, Columbia University, 630 West 168 th Street, New York, NY 10032.

Copyright (c) 1992 Society for Neuroscience $0270-6474 / 92 / 124800-08 \$ 05.00 / 0$
Axon growth occurs after nerve injury as well as during development, whether as the sustained regeneration seen in many peripheral neurons or as the abortive sprouting seen in the mammalian CNS. We have been using video microscopy to observe the early stages of such growth from transected Aplysia axons in culture. Massive numbers of filopodium-like protrusions (FLPs) form within minutes of transection, as the first step in vigorous growth from the axonal stump, and we thus saw this as a good protocol for studying the molecular events underlying the formation of filopodia. Unexpectedly, we find that many of the FLPs are microtubule-based rather than actin-based protrusions, revealing a previously unobserved ability of microtubules to participate in protrusion.

\section{Materials and Methods}

Cell culture. Neurons from buccal ganglia of juvenile Aplysia californica were cultured for video-enhanced contrast-differential interference contrast (VEC-DIC) microscopy as previously described (Goldberg and Burmeister, 1986). Cell bodies were extracted from ganglia with lengths of their original axons attached. Only neurons that had axons of at least several hundred micrometers in length were used for the experiments. They were cultured on glass coverslips that had been exposed to polylysine $(>300,000)$ and Aplysia hemolymph, the latter of which contains substrate-binding neurite growth-promoting activity (Burmeister et al., 1991). The culture medium was L-15 supplemented with salts (Schacher, 1985). For experiments involving the use of nominally $\mathrm{Ca}^{2+}$ free medium, the supplemented L-15 was replaced by an artificial sea water (Goldberg, 1988) shortly before the start of the experiment so as to allow the omission of $\mathrm{Ca}^{2+}$. Dishes with cells were left at room temperature for 15-26 hr before use in experiments. During this time, there was growth of neurites, predominantly from the ends of the axon stump.

Microscopy. VEC-DIC microscopy was performed as previously described (Goldberg and Burmeister, 1986; Burmeister et al., 1991). In a few experiments, we used a long working distance condenser (NA 0.65) rather than the usual short-working-distance condenser (NA 1.4) to allow greater access to the cells.

Neurons in culture were prepared for immunofluorescence microscopy by one of two methods. One method involved fixing the cells for $7.5 \mathrm{~min}$ with $0.25 \%$ glutaraldehyde $/ 400 \mathrm{~mm}$ sucrose $/ 100 \mathrm{~mm}$ cacodylic acid, followed by two rinses with artificial sea water containing sucrose (Burmeister et al., 1991) and 0.1\% Triton X-100 and then a $1 \mathrm{hr}$ incubation in $0.33 \mu \mathrm{M}$ rhodamine-phalloidin (to stain F-actin; Molecular Probes). The preparation was viewed by immunofluorescence microscopy and then processed as previously described (Burmeister et al., 1991) for visualizing microtubules by staining with fluoresceinated monoclonal antibody to $\beta$-tubulin. The other method involved replacing the supplemented L-15 medium with a slightly modified version of "buffer P" (composition, in mM: $103 \mathrm{~K}$ isethionate, $62 \mathrm{Na}$ isethionate, $18 \mathrm{KH}_{2} \mathrm{PO}_{4}, 123 \mathrm{KCl}, 10 \mathrm{MgCl}_{2}, 100 \mathrm{~K}$ L-aspartate, $18 \mathrm{~L}$-glutamine, 16 glycine, 132 taurine, 74 betaine, 4.5 glycerol, 1.2 glucose, 0.5 fructose, 1 ATP, 0.5 GTP, 1 EGTA, 0.1 phenylmethylsulfonyl fluoride, and 1 HEPES; $\mathrm{pH} 7.0$ ), a solution that had been formulated to match the composition of the axoplasm of squid, another marine mollusk (Morris and Lasek, 1982). This solution preserves cytoskeletal structures since it is $\mathrm{Ca}^{2+}$-free and contains ATP and GTP. Within a few minutes, this 

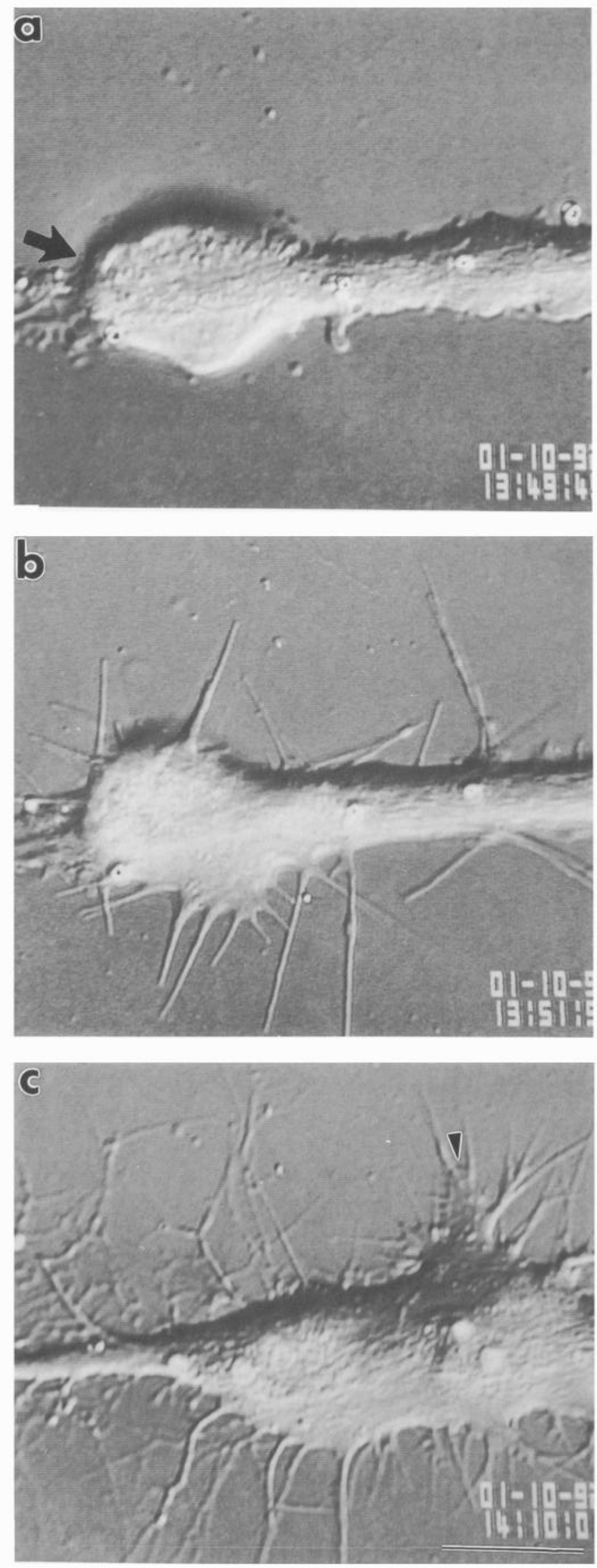

solution was replaced with modified buffer $\mathrm{P}$ containing $0.1 \%$ Triton $\mathrm{X}-100$ and $1 \%$ glutaraldehyde, which removed much or all of the plasma membrane and fixed the preparation. The preparation was processed to visualize microtubules by indirect immunofluorescence as described above. Comparatively low signal in the rhodamine channel demonstrated that fluorescence of FLP fibers was not due to autofluorescence.

Axotomy. Areas of axons were selected that had no neurites emanating from or running near them. These areas were devoid of filopodia. Transection of the axon was done while it was viewed through the microscope oculars at a magnification of $100 \times$. The cut was made with a glass micropipette whose tip was less than $0.5 \mu \mathrm{m}$, maneuvered by a hydraulically driven micromanipulator. All subsequent observations were of the axon segment that remained attached to the cell body, though FLPs formed on the detached segment as well.

At 5-6 min after axotomy, FLPs coming out of one side of the most distal $25 \mu \mathrm{m}$ of the axon were counted. Only those FLPs in focus at the level of the substrate were counted. By 10 min after axotomy, FLPs had appeared much farther from the sealed end of the axon, so total and transporting FLPs were counted on both sides of the most distal $50 \mu \mathrm{m}$ of the axon. Statistical comparisons were done by analysis of variance and the Bonferroni test for multiple groups (Wallenstein et al., 1980).

Chemicals. All three drugs that interact with the cytoskeleton were purchased from Sigma and stored at $4^{\circ} \mathrm{C}$ in stock dimethyl sulfoxide solutions, colcemid at $2.5 \mathrm{~mm}$, dihydrocytochalasin B at $5 \mathrm{~mm}$, and nocodazole at $10 \mathrm{~mm}$.

\section{Results}

The formation of large numbers of FLPs was triggered by transection of Aplysia axons in culture. Observation with high-resolution, high-magnification VEC-DIC microscopy suggested that the end of the axon sealed within a minute or two (our earliest observation), since the visible escape of cytoplasm and membranous organelles was staunched by the apparent formation of a delimitation. FLPs typically began appearing 4-7 min after transection and increased in number for several minutes (Fig. $1 a, b$; Table 1). They emerged both from the sealed end and along the length of the axon, typically being densely distributed up to $75 \mu \mathrm{m}$, and rarely found more than $150 \mu \mathrm{m}$, from the end. Veils of plasma membrane typically began moving between the FLPs along the sides of the axon 10-15 min after transection (Fig. 1c), some eventually giving rise to neurites. Thus, the formation of FLPs was the first morphological step in the growth of neurites after injury.

Elongation of individual FLPs was intermittent, but the instantaneous velocity of elongation was very high, averaging 1.6 $\pm 0.2 \mu \mathrm{m} / \mathrm{sec}$ (mean $\pm \mathrm{SEM} ; n=7$ ). Lengths of FLPs ranged up to roughly $50 \mu \mathrm{m}$. Many of the FLPs that formed in the first $10 \mathrm{~min}$ following axotomy grew out of blebs that had pushed out from the sides of the axon (Fig. 2). These blebs were structures whose size and, especially, shape changed rapidly. This morphological plasticity and the movements of the numerous membranous organelles within it imparted a churning quality to the bleb. Most of the organelles appeared to move randomly but some moved unidirectionally around the perimeter of the bleb.

Almost no FLPs formed when the axon was transected in medium containing no added $\mathrm{Ca}^{2+}$ rather than the $11 \mathrm{mM} \mathrm{Ca}^{2+}$ in normal medium (Table 1). (The altered medium did not contain $\mathrm{Ca}^{2+}$ chelator, so, presumably, small amounts of $\mathrm{Ca}^{2+}$

Figure 1. Formation of FLPs after axotomy. $a$, A few minutes after axotomy, the Aplysia axon in culture has apparently sealed (arrow) and has swollen at its new end. No FLPs have formed. $b$, Two minutes later, numerous FLPs have formed. $c$, Eighteen minutes later, more FLPs have formed and veils are protruding between some of them (arrowhead). Scale bar, $5 \mu \mathrm{m}$ for $a-c$. 


\begin{tabular}{|c|c|c|c|c|}
\hline & No drug & $0 \mathrm{Ca}^{2+}$ & Cytochalasin & Nocodazole \\
\hline $5-6 \mathrm{~min}$ & $2.9 \pm 0.7(17)$ & - & $3.3 \pm 0.6(21)$ & $0.2 \pm 0.2(6)^{a}$ \\
\hline $10 \mathrm{~min}$ & $69.3 \pm 5.1(10)$ & $3.3 \pm 1.4(4)^{b}$ & $34.1 \pm 5.9(7)^{b}$ & $29.5 \pm 9.3(4)^{c}$ \\
\hline Transporting & $4.1 \pm 1.3(10)$ & $0.0 \pm 0.0(4)$ & $10.1 \pm 2.2(8)^{d}$ & $0.0 \pm 0.0(6)$ \\
\hline \multicolumn{5}{|c|}{$\begin{array}{l}\text { Each entry is the mean }( \pm \text { SEM) number of FLPs, with the number of experiments in parentheses. See Materials and } \\
\text { Methods for methods of counting FLPs, and note that the counts were made from larger lengths at } 10 \text { min than at } 5 \text { - } \\
6 \text { min. }\end{array}$} \\
\hline $\begin{array}{l}\text { a Significantly d } \\
\text { ' Significantly d } \\
\text { ' Significantly di } \\
{ }^{\circ} \text { Significantly d }\end{array}$ & $\begin{array}{l}\text { from "cytochalasin, } \\
\text { from "No drug," } p \\
\text { from "No drug," } p \\
\text { from "No drug," } p\end{array}$ & $\begin{array}{l}0.05 \\
001 \\
01\end{array}$ & & \\
\hline
\end{tabular}

were present.) The rare FLP in this medium did not elongate, remaining instead a short, fat, flaccid projection (Fig. 3). Restoration of the normal concentration of $\left[\mathrm{Ca}^{2+}\right]_{0}(4 \mathrm{~min}$ after transection in one experiment and 9 or $10 \mathrm{~min}$ in three others) did not cause substantial numbers of FLPs to form, suggesting that influx of $\mathrm{Ca}^{2+}$ through the cut end was essential for their formation.

Some of the FLPs that formed shortly after axotomy displayed rapid transport of swellings (Fig. 4, Table 1). Some unequivocally displayed transport virtually as soon as they started growing (Fig. 5). Transported swellings ranged in apparent diameter from about $0.2 \mu \mathrm{m}$ (detectable objects smaller than this are inflated to this apparent diameter by VEC-DIC microscopy) to about $1.7 \mu \mathrm{m}$. Some swellings were probably clumps of membranous organelles. Others may have been single membranous organelles because, in occasional favorable situations, we could see what appeared to be a single organelle transported from an FLP into the axon (Fig. 6). Bidirectional movement was seen in many FLPs that displayed transport. The instantaneous velocity of transport of swellings moving toward the tip of the FLP was $1.8 \pm 0.1 \mu \mathrm{m} / \mathrm{sec}(n=10$; each measurement was made in a different FLP) and of swellings moving in the opposite direction was $2.1 \pm 0.2(n=10)$. Most of the transporting FLPs displayed transport along much of their length; swellings were even sometimes seen being transported all the way to the tip, or from the tip, of growing FLPs (Fig. 5).

Fast transport of membranous organelles in animal cells has been convincingly demonstrated to occur along microtubules (Schnapp et al., 1985), suggesting the presence of microtubules in the transporting FLPs. However, filopodia generally do not contain microtubules, being rich in actin filaments instead (Letourneau and Ressler, 1983; Tosney and Wessells, 1983). Also, there is evidence that membranous organelles can move along actin filaments in axoplasm (Kuznetsov et al., 1992), and a recent report suggested that actin filaments can power the transport of swellings in growth cone filopodia (Sheetz et al., 1990). In occasional optically favorable situations, VEC-DIC microscopy revealed a fiber extending from the living axon into an FLP and even bending or moving from side to side. Such flexibility is similar to that observed for microtubules in living growth cones (Tanaka and Kirschner, 1991), but the identity of such fibers could not be ascertained with VEC-DIC microscopy. Experiments were therefore done to determine which cytoskeletal element was responsible for the observed transport.

Cytochalasin, which blocks the polymerization of actin filaments, did not inhibit the formation of transporting FLPs, but nocodazole and colcemid, which block the polymerization of microtubules, did (Table 1). In the presence of $20 \mu \mathrm{M}(9.6 \mu \mathrm{g} /$ ml) dihydrocytochalasin B (a high concentration), FLPs began appearing as soon after transection as without drug, as judged by their presence 5-6 min after transection (Table 1 ). The overall number of FLPs present $10 \mathrm{~min}$ after transection in dihydrocytochalasin was only half of that seen without drug, but the number of FLPs displaying transport was larger (Table 1). The results were quite different when transection was done in the presence of $2 \mu \mathrm{M}(0.6 \mu \mathrm{g} / \mathrm{ml})$ nocodazole (a modest concentration) rather than dihydrocytochalasin. There was a delay in the formation of FLPs. In only one of the six axons studied had any FLPs appeared by 5-6 min after axotomy, and only a single FLP had appeared in that case (Table 1). By 10 min after axotomy there were still markedly fewer FLPs than seen without drug - on average, less than half (Table 1)-but many FLPs had formed in all of the axons by 17-20 min after axotomy (data not shown). No transporting FLPs were observed at any time after transection in nocodazole in any of the axons (Table 1). Transporting FLPs were also not observed in any of three axons transected in $20 \mu \mathrm{M}(7.4 \mu \mathrm{g} / \mathrm{ml})$ colcemid (data not shown). Also, addition of $5 \mu \mathrm{M}(1.5 \mu \mathrm{g} / \mathrm{ml})$ nocodazole to drug-free medium after axotomy caused rapid beading of newly formed transporting FLPs, though transporting FLPs that were several minutes old continued to display transport for many minutes in the presence of nocodazole (data not shown). No FLPs formed in the simultaneous presence of $20 \mu \mathrm{M}$ dihydrocytochalasin and 2 $\mu \mathrm{M}$ nocodazole.

We obtained a direct demonstration of the presence of $\mathrm{mi}$ crotubules in some FLPs by doing immunofluorescence microscopy with monoclonal antibody to tubulin. Figure 7 shows FLPs that grew out of an axon near the site of transection, stained with this antibody (Fig. 7a) but not with fluorescent phalloidin (Fig. $7 b$ ), which labels F-actin and brightly stains filopodia of growth cones (Fig. $7 b$, inset). We did additional experiments in which we permeabilized while fixing to ensure that the fluorescence in FLPs emanated from tubulin polymer rather than monomer. It was clear that the plasma membrane was solubilized by our procedure, since cytoplasm was seen to escape from the axon and multiple thin fibers could be seen with VEC-DIC microscopy in cases where there had been a thick FLP (Fig. 8). Eighteen of 21 transporting FLPs observed in five axons, as well as numerous FLPs in which transport had not been noted, were found to have fibers that stained with the monoclonal antibody to tubulin (Fig. 9).

\section{Discussion}

It is clear that a substantial number of the FLPs that form soon after axotomy contain microtubules, and it seems likely that microtubules drive the formation of some or all of the trans- 

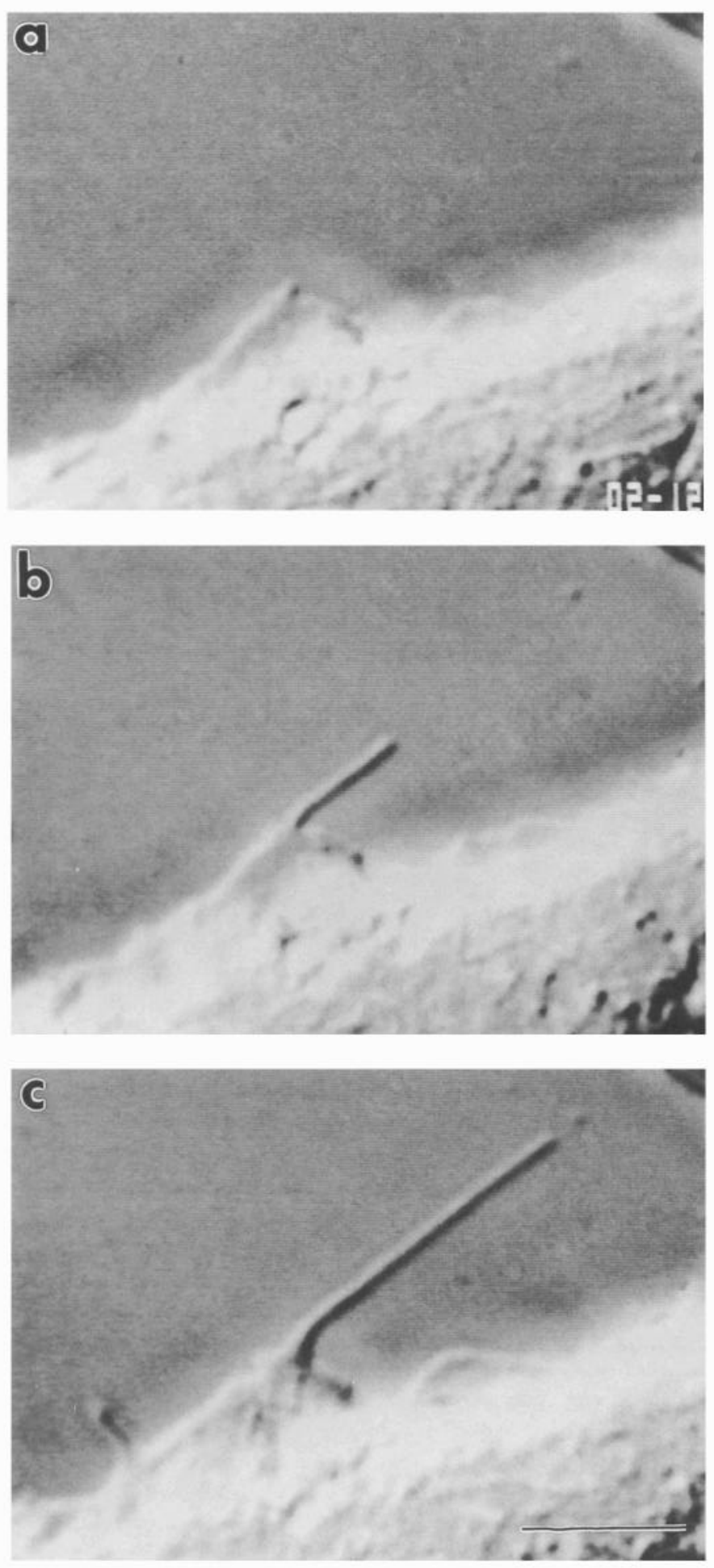

Figure 2. Growth of an FLP from a bleb. $a$, The nub of an FLP has appeared on a bleb that erupted from the side of the transected axon. $b$, Three seconds later the nub has grown into an FLP. $c$, After another $5 \mathrm{sec}$ the FLP has elongated more, a new FLP has emerged from the base of the bleb, and a new bleb has formed to the right. Scale bar, 5 $\mu \mathrm{m}$ for $a-c$.

porting FLPs rather than entering FLPs whose formation depended on actin filaments (see below). Because filopodia are invariably described as having a cytoskeleton of actin filaments, we prefer to refer to all of the "filopodia" that form soon after
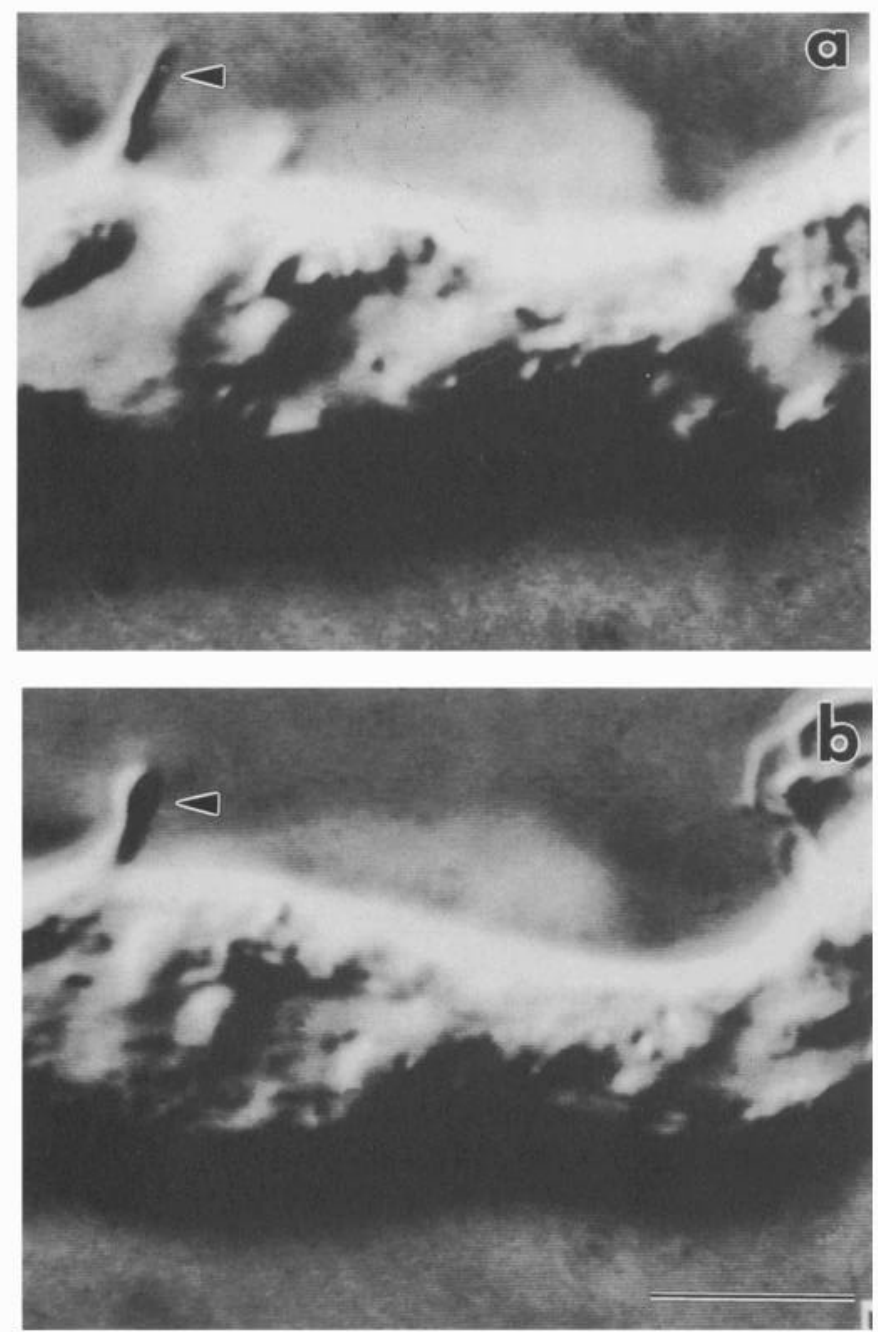

Figure 3. Block of formation and lengthening of FLPs by removal of $\mathrm{Ca}^{2+}$ from the extracellular medium. $a$, Several minutes after the transection of an axon in medium containing no added $\mathrm{Ca}^{2+}$, only a single short FLP (arrowhead) has formed. The sealed end of the axon is slightly to the right of the displayed field. $b$, Twelve minutes later, that FLP has not grown and no others have formed. Scale bar, $5 \mu \mathrm{m}$ for $a$ and $b$.

axotomy as FLPs. The important finding is that microtubules can apparently power the rapid growth of long protrusions from axons. This has not been shown previously, though microtubuledriven protrusions are well known in certain other types of cells, for example, in the form of cilia. That neurite growth can be driven by microtubules was established in culture by Letourneau and colleagues (Marsh and Letourneau, 1984; Letourneau et al., 1987) and in situ by Bentley and Toroian-Raymond (1986), who demonstrated growth in the presence of cytochalasin. The present results are another manifestation of the ability of the push of microtubules to advance the margins of the axon, the difference here being that we have described the formation of multiple thin protrusions that grow hundreds of times faster than cytochalasin-treated neurites.

Several pieces of evidence attest to the presence of microtubules in some FLPs. First, transport of swellings in FLPs had a velocity similar to microtubule-based fast transport in the axon. The measured net velocity of anterograde fast axonal transport in Aplysia axons is $1.5 \mu \mathrm{m} / \mathrm{sec}$ (Goldberg et al., 1978), and the instantaneous velocities for the two directions of transport in 

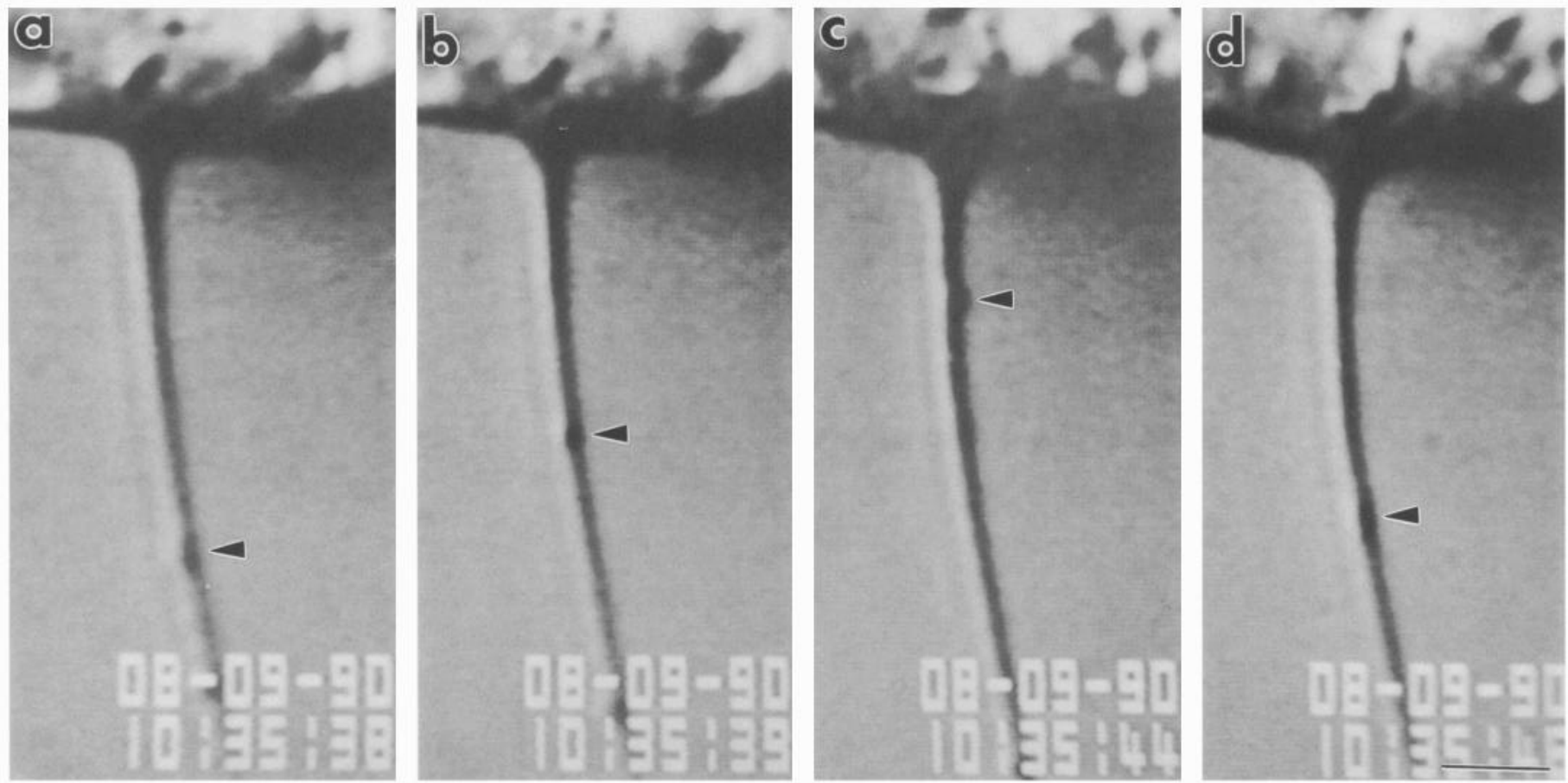

Figure 4. Bidirectional transport of swellings in an FLP. $a$ and $b$, A swelling (arrowheads) moves toward the axon in the interval between $a$ and $b$. $c$ and $d$, Several seconds later, another swelling (arrowheads) moves in the opposite direction in the interval between $c$ and $d$. Scale bar, $2 \mu \mathrm{m}$ for $a-d$.

FLPs were 1.8 and $2.1 \mu \mathrm{m} / \mathrm{sec}$. Second, no transporting FLPs were formed in the presence of nocodazole or colcemid, drugs that block microtubule polymerization and cause depolymerization of dynamic microtubules, and nocodazole had destructive effects on some transporting FLPs that had formed in its absence. Lastly, fibers revealed in FLPs, including transporting FLPs, by membrane solubilization stained with a monoclonal antibody to tubulin. The microtubule-based FLPs probably start forming sooner after axotomy than the actin-based FLPs. A much higher percentage of the initial FLPs than of the FLPs present later displayed transport (data not shown). Also, FLPs formed as quickly with as without dihydrocytochalasin B present, but their formation was delayed by the presence of nocodazole.

While the data clearly demonstrate the presence of microtubules in some transporting FLPs, we do not know if all the FLPs that contain microtubules display transport. The results with nocodazole (Table 1) suggest that half of the FLPs formed within $10 \mathrm{~min}$ of axotomy have microtubules, and immunofluorescence showed many microtubules in FLPs. In contrast, less than $5 \%$ of the FLPs were scored as displaying transport. However, this may be an underestimate of transporting FLPs, since
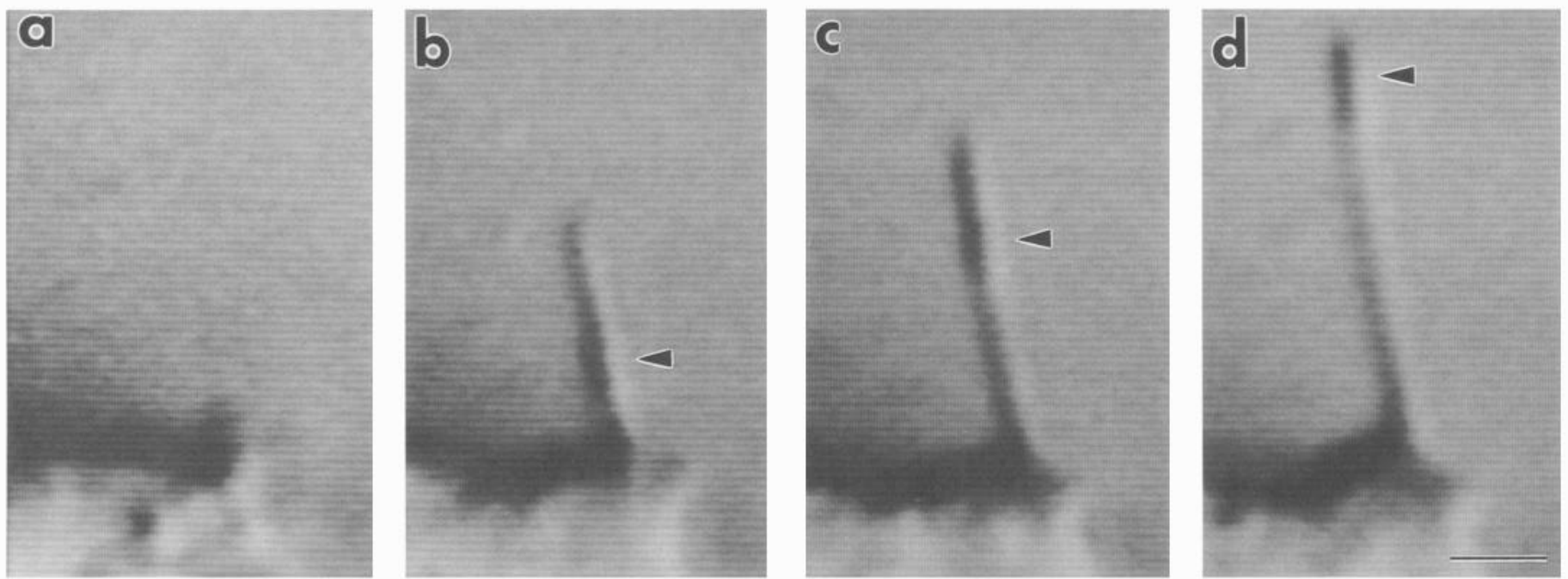

Figure 5. Transport of swelling in a newly formed FLP. $a$, The nub of an FLP has appeared on a bleb. $b$, Twenty-four seconds later the nub has lengthened into an FLP and a sausage-shaped swelling (arrowhead) has just moved into the FLP. $c$, Two seconds later the FLP has grown more and the swelling has moved farther. $d$, Two seconds later the FLP has grown more and the swelling has been transported to the tip of the FLP. Scale bar, $1 \mu \mathrm{m}$ for $a-d$. 

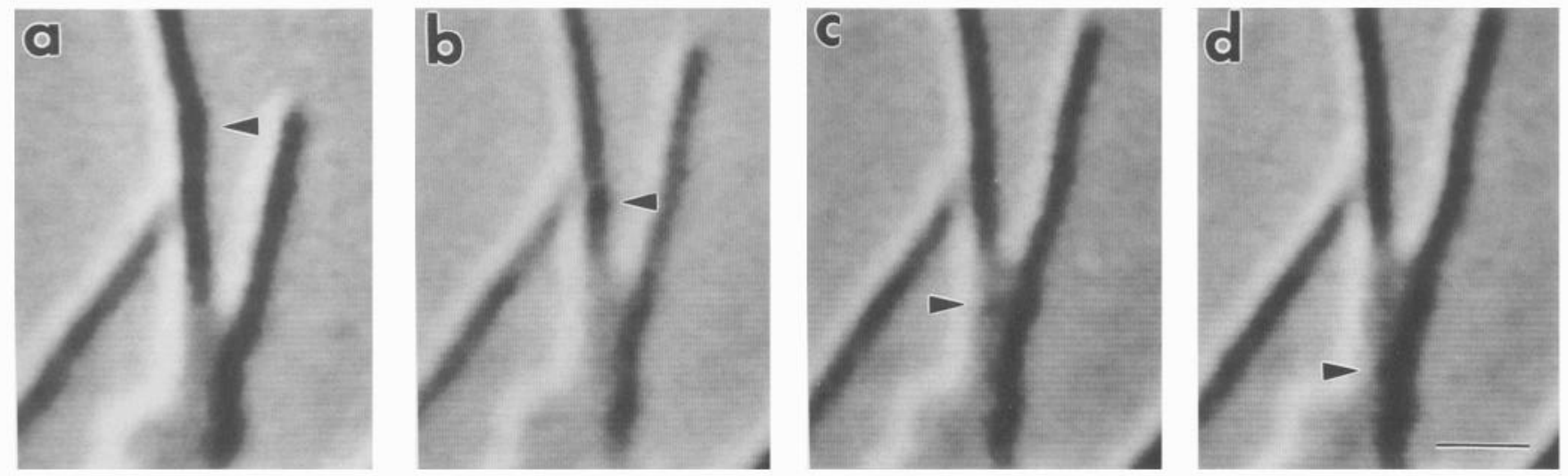

Figure 6. Transport of an apparent single organelle (arrowheads) from an FLP into a bleb. The intervals between the panels are $670 \mathrm{msec}$ between $a$ and $b, 835 \mathrm{msec}$ between $b$ and $c$, and $465 \mathrm{msec}$ between $c$ and $d$. Scale bar, $1 \mu \mathrm{m}$ for $a-d$.

each microscope field was observed for no more than $30 \mathrm{sec}$ in counting transporting FLPs.

Without observing microtubules in the living axon while FLPs are forming, we cannot be certain that microtubules drive the formation of some of the FLPs rather than entering after formation. However, several findings, when taken together, strongly suggest this to be true. In some instances, transport of swellings was observed almost immediately upon formation of an FLP. In other instances, transport was observed at the tip of growing FLPs, implying the extension of microtubules to the tip. Acutely added nocodazole caused beading and cessation of growth of some newly formed FLPs. Also, if all of the FLPs were actin driven, one would not have expected the delay in appearance of FLPs in the presence of nocodazole; FLPs should have formed as rapidly but not have displayed transport. In addition, immunofluorescence microscopy showed that FLPs that stained with an antibody to tubulin did not stain with phalloidin, which binds to F-actin. Lastly, it is highly unlikely that any of the numerous FLPs that formed in the presence of $20 \mu \mathrm{M}$ dihydrocytochalasin B were actin driven, as this concentration of the drug should block actin polymerization.

A recent study reported bidirectional transport of refractile aggregates in filopodia of cultured mouse cerebral neurons and concluded that this was powered by actin filaments, partly because the movements were not halted by nocodazole (Sheetz et al., 1990). Because, in our study, some of the transporting FLPs later shown to contain microtubules were not readily distinguishable in VEC-DIC microscopy from traditional filopodia, and since transport of swellings in older FLPs could persist for a considerable time in the presence of nocodazole, such a conclusion seems tenuous without more direct evidence.

What is the mechanism of formation of microtubule-containing FLPs? Sliding of microtubules along one another or lengthening of microtubules by polymerization are the likely possibilities. The facts that the rate of elongation of the FLP is virtually identical to the rate of fast axonal transport but is more than an order of magnitude greater than most reported rates of microtubule polymerization in vivo and in vitro (Chen and Schliwa, 1990; Tanaka and Kirschner, 1991) suggest sliding as the mechanism. However, there was a recent report of lengthening of microtubules in vivo at a rate of $1.6 \mu \mathrm{m} / \mathrm{sec}$ (Chen and Schliwa, 1990), and no transporting FLPs formed in the presence of nocodazole or colcemid, drugs that block polymerization, so we cannot now distinguish between these possibilities.

Whichever is the mechanism, we suggest that it is enabled by localized weakening of the cortical layer of actin filaments. It has been suggested that tension exerted by the actin network of the growth cone inhibits the elongation of microtubules (Joshi et al., 1985), and it has been shown that weakening of this network with cytochalasin promotes microtubule advance (Forscher and Smith, 1988; Burmeister et al., 1991). The emergence of blebs after axotomy suggests local cortical weakening (Albrect-Buehler, 1987). The apparent dependence of the formation of the FLPs on $\mathrm{Ca}^{2+}$ influx, which is known to occur as a result of axotomy (Strautman et al., 1990) and to be particularly destructive of actin filaments (Lankford and Letourneau,
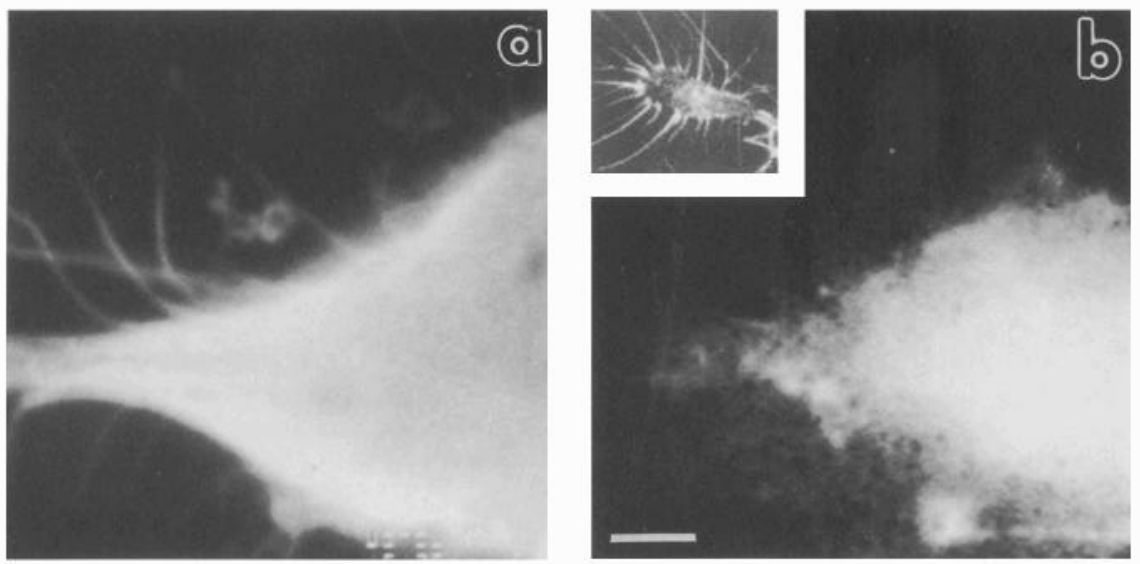

Figure 7. Staining of some FLPs with antibody to tubulin but not phalloidin. $a$, Immunofluorescence micrograph shows four FLPs projecting from the upper surface of a recently transected axon that was fixed and stained with monoclonal antibody to tubulin. (The stained fiber running horizontally across three of the FLPs is not itself an FLP, but rather a preexisting thin neurite.) $b$, Staining with fluorescent phalloidin does not reveal F-actin in the FLPs, though filopodia of a growth cone are stained (inset). Scale bar, $5 \mu \mathrm{m}$ for $a$ and $b ; 20 \mu \mathrm{m}$ for inset. 
Figure 8. Exposure of internal fibers of FLPs by removal of plasma membrane. $a$, A pair of thick FLPs is shown before removal of the plasma membrane and fixation. $b$, Treatment with Triton and glutaraldehyde removes the plasma membrane, as evidenced by the unmasking of multiple fibers in each FLP. Scale bar, $5 \mu \mathrm{m}$ for $a$ and $b$.
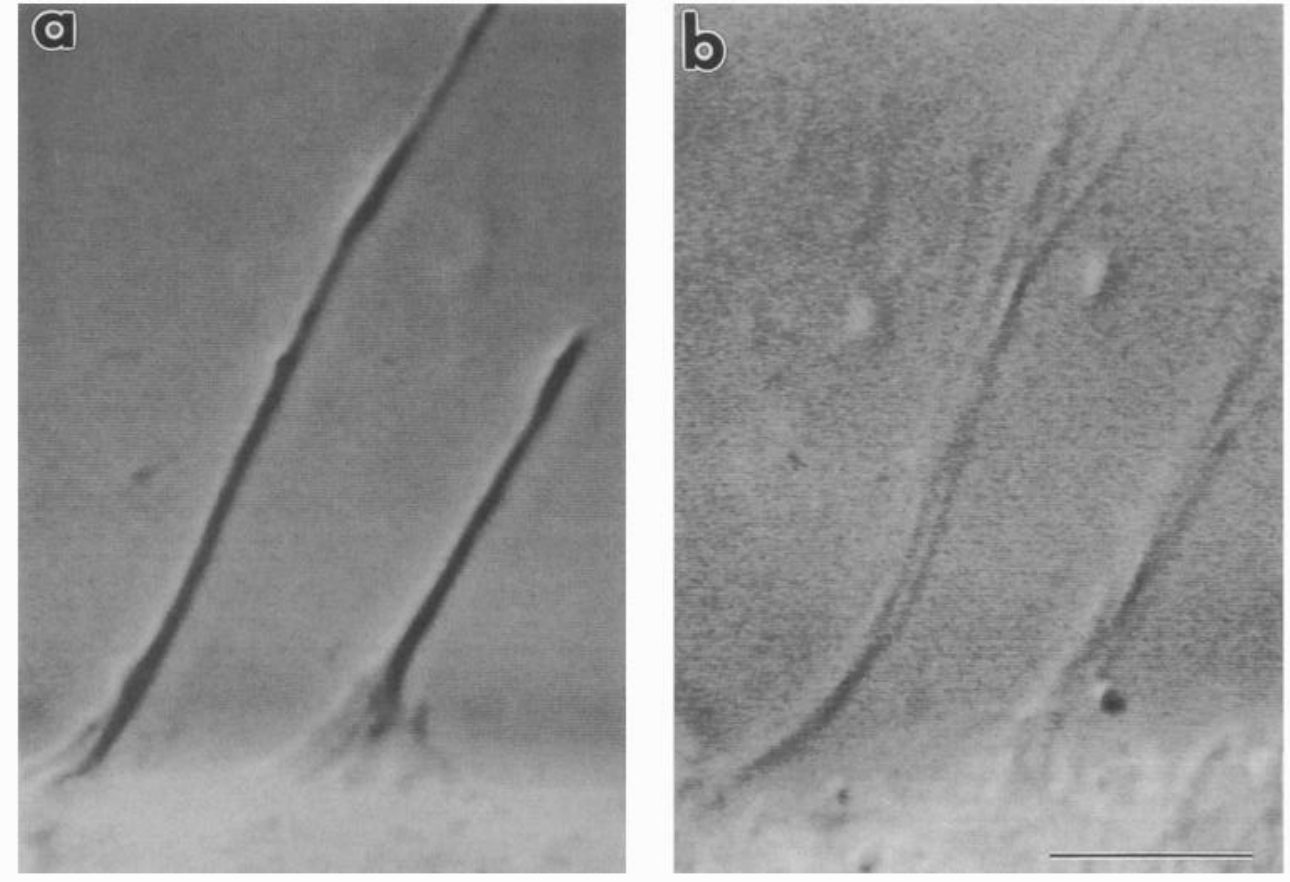

1989), is also consistent with cortical weakening, as is the increase in the number of transporting FLPs when transection was done in the presence of dihydrocytochalasin B.

Whether microtubule-based protrusions form in situations other than after axotomy remains to be seen. Studies of axon growth of grasshopper pioneer neurons in vivo have demonstrated that a filopodium can mature into an axonal branch (O'Connor et al., 1990). The first step in this maturation is the entry of a microtubule (Sabry et al., 1991). Thus, FLPs that have microtubules from the outset might be prestabilized, which could be useful in situations where rapid formation of a neuritic branch was needed. One interesting situation at which to look is the formation of a collateral off the shaft of a growing axon at a point proximal to the growth cone ("back-branching"), which may be an important mechanism of arborization in vivo (Harris et al., 1987; O'Leary and Terashima, 1988). Such branches do not seem to have conventional growth cones.

\section{References}

Albrect-Buehler G (1987) Role of cortical tension in fibroblast shape and movement. Cell Motil Cytoskel 7:54-67.

Aletta JM, Greene LA (1988) Growth cone configuration and advance: a time-lapse study using video-enhanced differential interference contrast microscopy. J Neurosci 8:1425-1435.

Bentley D, Toroian-Raymond A (1986) Disoriented pathfinding by pioneer neurone growth cones deprived of filopodia by cytochalasin treatment. Nature 323:712-715.

Burmeister DW, Rivas RJ, Goldberg DJ (1991) Substrate-bound factors stimulate engorgement of growth cone lamellipodia during neurite elongation. Cell Motil Cytoskel 19:255-268.
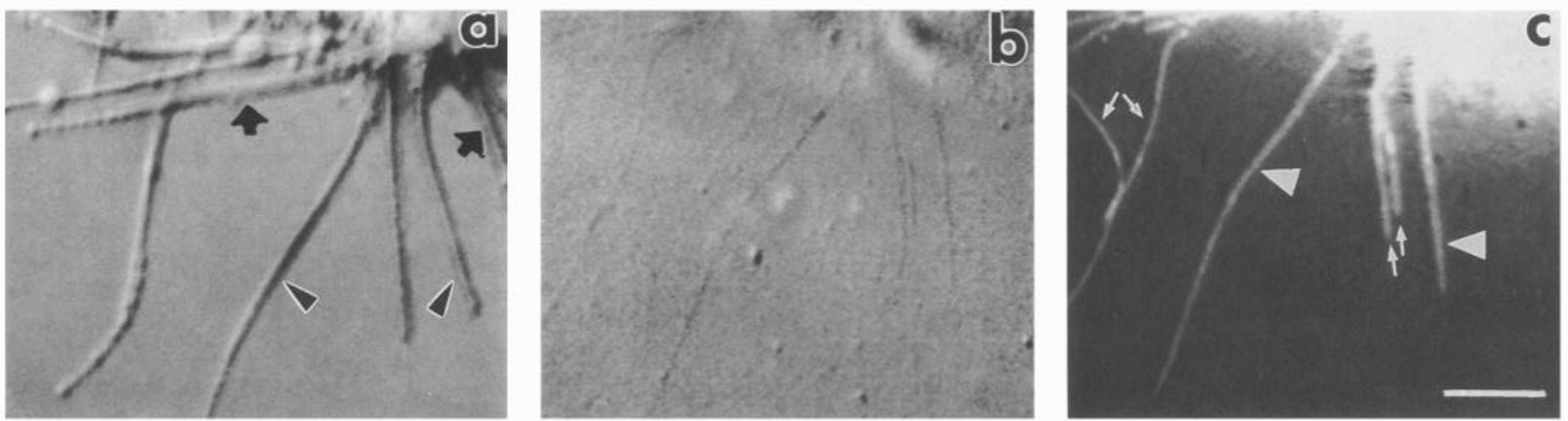

Figure 9. Fibers in FLPs stain with an antibody to tubulin. $a$, Several FLPs on the side of an axon (top of field) are shown after the addition of modified buffer $\mathrm{P}$ (which has caused some beading) but before the addition of Triton and glutaraldehyde. Transport of swellings was occurring in at least two of the FLPs (arrowheads) before the addition of buffer P. $b$, After the addition of Triton and glutaraldehyde, fibers can be seen corresponding to some of the FLPs in $c$. Some of the fibers have moved slightly during the exchange of solutions and the field has been moved slightly. $c$, These fibers stain with a monoclonal antibody to $\beta$-tubulin, as detected by immunofluorescence. Both of the FLPs in which transport was observed before fixation contained fibers that stain with the antibody (arrowheads). Removal of the plasma membrane has apparently revealed the presence of two fibers (which have separated) in each of two other FLPs (arrows). Fibers were not revealed by VEC-DIC or fluorescence microscopy in two other FLPs (arrows in $a$ ). The brightly stained axon is at the $t$ op. Scale bar, $5 \mu \mathrm{m}$ for $a-c$. 
Chen Y-T, Schliwa M (1990) Direct observation of microtubule dynamics in Reticulomyxa: unusually rapid length changes and microtubule sliding. Cell Motil Cytoskel 17:214-226.

Forscher P, Smith SJ (1988) Actions of cytochalasins on the organization of actin filaments and microtubules in a neuronal growth cone. J Cell Biol 107:1505-1516.

Goldberg DJ (1988) Local role of $\mathrm{Ca}^{2+}$ in formation of veils in growth cones. J Neurosci 8:2596-2605.

Goldberg DJ, Burmeister DW (1986) Stages in axon formation: observations of growth of Aplysia axons in culture using video-enhanced contrast-differential interference contrast microscopy. J Cell Biol 103; 1921-1931.

Goldberg DJ, Burmeister DW (1989) Looking into growth cones. Trends Neurosci 12:503-506.

Goldberg DJ, Schwartz JH, Sherbany AA (1978) Kinetic properties of normal and perturbed axonal transport of serotonin in a single identified axon. J Physiol (Lond) 281:559-579.

Harris WA, Holt CE, Bonhoeffer F (1987) Retinal axons with and without their somata growing to and arborizing in the tectum of Xenopus embryos: a time-lapse video study of single fibres in vivo. Development 101:123-133.

Joshi HC, Chu D, Buxbaum RE, Heidemann SR (1985) Tension and compression in the cytoskeleton of PC12 neurites. J Cell Biol 101: 697-705.

Kuznetsov SA, Langford GM, Weiss DG (1992) Actin-dependent organclle movement in squid axoplasm. Naturc 356:722-725.

Lankford KL, Letourneau PC (1989) Evidence that calcium may control neurite outgrowth by regulating the stability of actin filaments. J Cell Biol 109:1229-1243.

Letourneau PC, Ressler AH (1983) Differences in the organization of actin in the growth cones compared with the neurites of cultured neurons from chick embryos. J Cell Biol 97:963-973.

Letourneau PC, Shattuck TA, Ressler AH (1987) "Pull" and "push" in neurite elongation: observations on the effects of different concentrations of cytochalasin B and taxol. Cell Motil Cytoskel 8:193-209.

Marsh L, Letourneau PC (1984) Growth of neurites without filopodial or lamellipodial activity in the presence of cytochalasin B. J Cell Biol 99:2041-2047.

Morris JR, Lasek RJ (1982) Stable polymers of the axonal cytoskeleton: the axoplasmic ghost. J Cell Biol 92:192-198.

O'Connor TP, Duerr JS, Bentley D (1990) Pioneer growth cone steering decisions mediated by single filopodial contacts in situ. J Neurosci 10:3935-3946.

O'Leary DDM, Terashima T (1988) Cortical axons branch to multiple subcortical targets by interstitial axon budding: implications for target recognition and "waiting periods." Neuron 1:901-910.

Sabry JH, O'Connor TP, Evans L, Toroian-Raymond A, Kirschner M, Bentley D (1991) Microtubule behavior during guidance of pioneer neuron growth cones in situ. J Cell Biol 115:381-395.

Schacher S (1985) Differential synapse formation and neurite outgrowth at two branches of the metacerebral cell of Aplysia in dissociated cell culture. J Neurosci 5:2028-2034.

Schnapp BJ, Vale RD, Sheetz MP, Reese TS (1985) Single microtubules from squid axoplasm support bidirectional movement of organelles. Cell 40:449-454.

Sheetz MP, Baumrind NL, Wayne DB, Pearlman AL (1990) Concentration of membrane antigens by forward transport and trapping in neuronal growth cones. Cell 61:231-241.

Smith SJ (1988) Neuronal cytomechanics: the actin-based motility of growth cones. Science 242:708-715.

Strautman AF, Cork RJ, Robinson KR (1990) The distribution of free calcium in transected spinal axons and its modulation by applied electrical fields. J Neurosci 10:3564-3575.

Tanaka EM, Kirschner MW (1991) Microtubule behavior in the growth cones of living neurons during axon elongation. J Cell Biol 115:345363.

Tosney KW, Wessells NK (1983) Neuronal motility: the ultrastructure of veils and microspikes correlates with their motile activities. J Cell Sci 61:389-411.

Wallenstein S, Zucker CL, Fleiss JL (1980) Some statistical methods useful in circulation research. Circ Res 47:1-9. 